\title{
Érosion-corrosion dans les turbines à vapeur humide. Expériences acquises et dispositions prises
}

\author{
Erosion-corrosion in damp-steam turbines. \\ Experience acquired and steps taken
}

\author{
H. Keller et A. W. Schmitz \\ Kraftwerk Union (KWU), Mülheim a. d. Ruhr, RFA
}

Différentes publications exposent, - en particulier pour un courant d'eau pure -, les bases des processus électro-chimique et thermo-hydraulique qui amènent à l'érosion-corrosion.

La construction et le fonctionnement de turbines exploitées en vapeur humide dans les centrales nucléaires à réacteur refroidi par eau légère soulevaient quelques problèmes spéciaux supplémentaires. Ceux-ci provenaient de l'expansion de la vapeur motrice dans l'étendue de son conditionnement biphasé et de l'écoulement entre les composants fixes et ceux toumants des machines.

Ces difficultés obligeaient les ingénieurs à concevoir des modèles de pensée pour saisir les particularités de leurs projets de construction. Les méthodes de calcul qui s'en ensuivaient fournissaient des éléments quantitatifs appuyés sur les expériences d'exploitation de la première génération de turbines mues par la vapeur humide. Une méthode de calcul de ce genre qui répond à l'attente de la KWU est décrite dans l'exposé [1]. Entretemps, des expériences complémentaires acquises avec une génération ultérieure de turbines pour vapeur humide sont devenues disponibles. Dans l'ensemble, on peut dire que cette méthode de pronostiquer s'est avérée bien valable pour le choix de détails de conception, de paramètres thermodynamiques et de matières premières et que le nombre d'abrasions graves s'est réduit très fortement. Cependant, les révisions de groupes turbo-alternateurs révèlent en ce moment encore toujours des surprises. Il y a des cas où malgré le résultat du pronostic calculé qui annonçait un grand risque d'abrasion, de bonnes expériences spéciales incitaient à faire abstraction de mesures protectrices alors qu'on constatait finalement que dans le cas concret, la turbine s'en tenait à l'indication foumie par le calcul et ignorait les expériences acquises jusque-là. Enfin, il se manifeste évidemment aussi le cas où un potentiel de danger surgit là où l'on ne le présumait pas.
Dans ce qui suit, la philosophie de calcul déjà décrite en détail autrefois est exposée d'une manière succincte afin d'en référer pour décrire des expériences et des dispositions complémentaires.

D'après cette philosophie, le taux d'abrasion aux endroits où celle-ci locale est maximale se calcule par la formule :

$$
\dot{s}=f(\vartheta) . f(x) \cdot K_{c} \cdot c-K_{s} \mathrm{~mm} / 10000 \mathrm{~h} .
$$

$f(\vartheta)$ est une fonction de la température de saturation.

Par contre, $f(x)$ est une fonction de l'humidité. Ces deux grandeurs n'ont pas de dimensions.

L'influence de ces deux paramètres ressort du diagramme de Mollier (fig. 1) qui montre l'allure de la détente dans les turbines de constructions typiques et l'étendue d'érosion-corrosion partielle. Cette étendue est située essentiellement entre $100^{\circ} \mathrm{C}$ et $230^{\circ} \mathrm{C}$ avec un maximum à $180^{\circ} \mathrm{C}(10$ bar $)$ et s'applique également jusque dans la zone de très faible humidité. $f(\vartheta)$ se lit en particulier sur la figure 2. Pour l'influence de l'humidité, on pose

$$
f(x)=\sqrt{1-x}
$$

L'influence de la géométrie du courant est prise en considération par la grandeur $K_{c}$ qui est de fait un rapport de vitesses $(\mathrm{mm} / 10000 \mathrm{~h})(\mathrm{m} / \mathrm{s})$ et d'autant plus grande qu'à proximité de la paroi la composante normale à celle-ci de la vitesse du courant principal ou de celui secondaire est plus importante. La figure 3 représente des configurations typiques d'écoulement pour ces composantes normales. La figure 4 indique des données quantitatives pour $K_{c}$ dont certaines s'assortissent surtout à l'application dans les turbines à vapeur, notamment aux joints a'assemblage ( $\left.\mathrm{n}^{\circ} 14\right)$, aux labyrinthes d'occlusion d'arbres en rotation $\left(n^{\circ} 15\right)$ et de cerclage des aubes $\left(n^{\circ} 16\right)$.

L'estimation du taux d'érosion tient compte aussi de la vitesse $\mathrm{cm} / \mathrm{s}$ du courant principal (cas 1 à 13). 


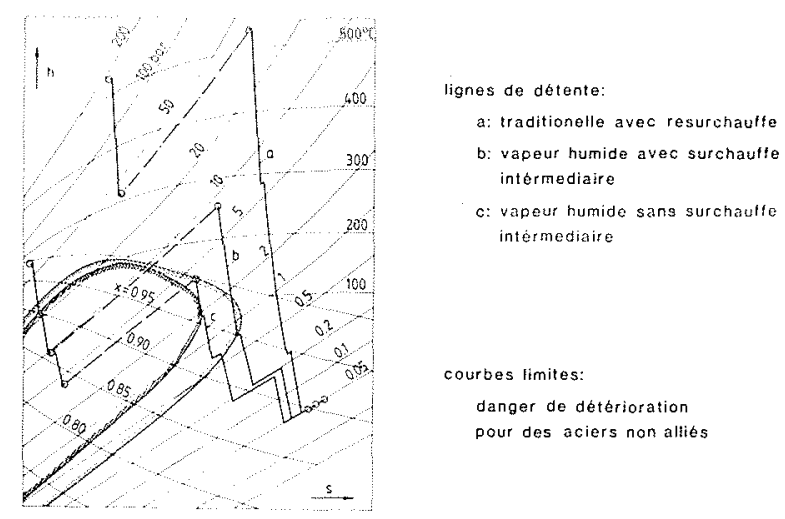

Figure 1 - Erosion-corrosion dans les turbines à vapeur saturée. Diagramme de Mollier, lignes de détente, zone d'attaque.

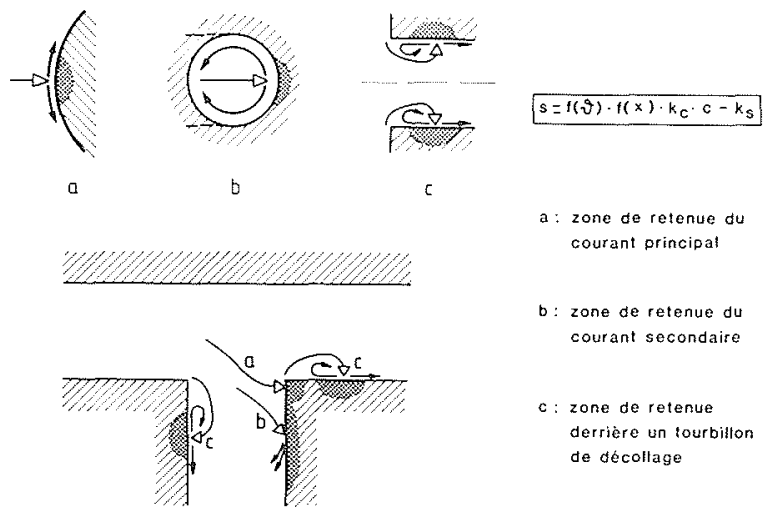

Figure 3 - Erosion-corrosion dans les turbines à vapeur saturée. Configurations de courant pour l'enlèvement typique.
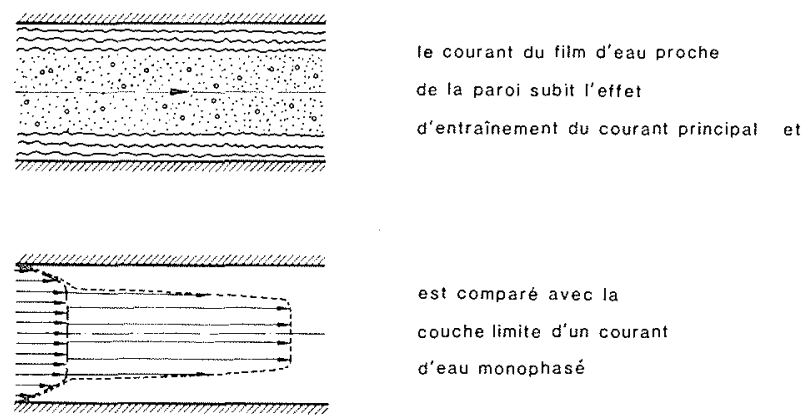

est comparé avec la

couche limite d'un courant

d'eau monophasé

Figure 5 - Erosion-corrosion dans les turbines à vapeur saturée. Analogie des courants biphasés et monophasés.

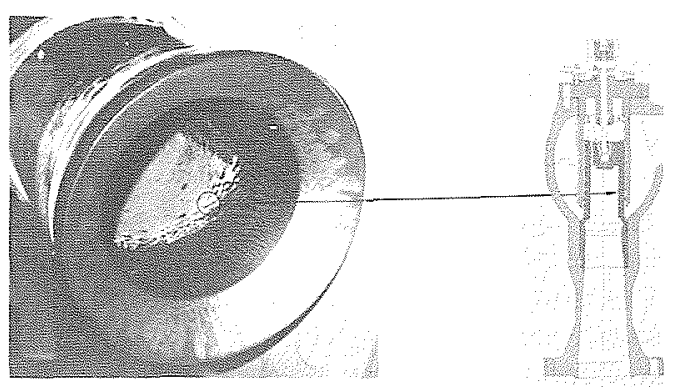

Figure 7. - Erosion-corrosion dans les turbines à vapeur saturée. Enlèvements à l'intérieur d'une cloche de soupape de réglage.

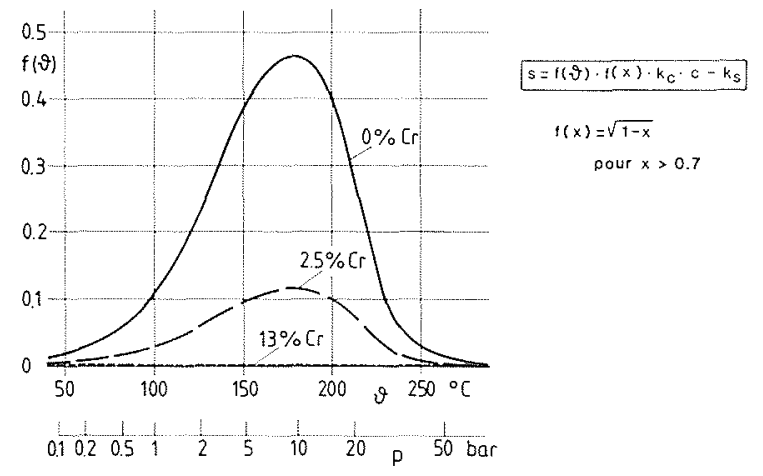

Figure 2 - Erosion-corrosion dans les turbines à vapeur saturée. Influence de la température et de la densité de la phase vapeur.

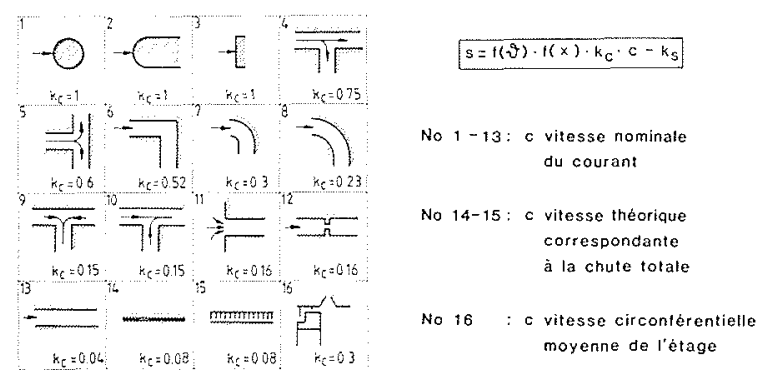

Figure 4 - Erosion-corrosion dans les turbines à vapeur saturée. Influence de la géométrie.

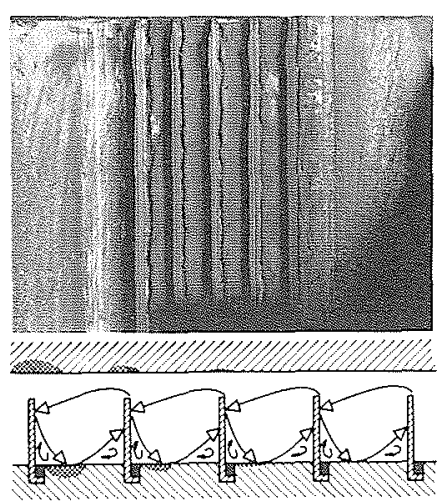
les enlèvements augmentent vers le bout des chicanages en fonction de l'augmentation de vilesse
contre - mesures: matériau de base en $2.5 \%$ de chrome of garniture protectrice pliée

Figure 6. - Erosion-corrosion dans les turbines à vapeur saturée. Configuration de courants et enlèvements aux chicanages.

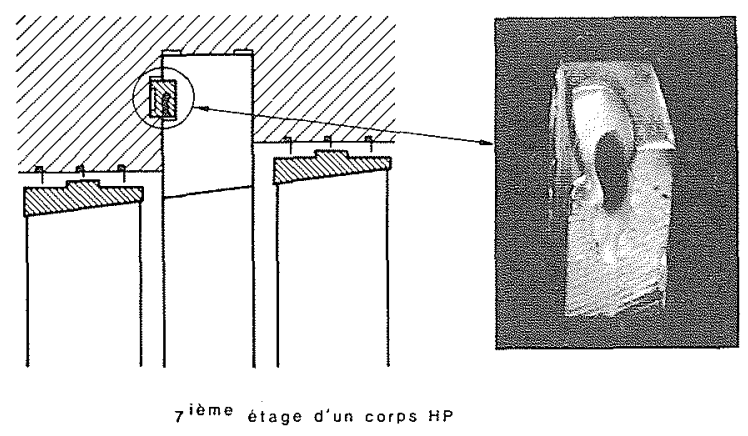

Figure 8 - Erosion-corrosion dans les turbines à vapeur saturée. Chose curieuse : Trace du légendaire ver de fer. 
Pour les cas (14) et (15) de la figure 4, la vitesse considérée est celle correspondant sans pertes à la chute totale de pression; pour le cas (16), la vitesse est définie comme celle périphérique moyenne de l'étage.

Enfin, l'énonciation $K_{s}=1 \mathrm{~mm} / 10000 \mathrm{~h}$ a égard à une valeur de seuil en dessous de laquelle on ne s'attend plus aux abrasions, ce en raison de la formation d'une couche protectrice suffisante de recouvrement.

Le taux local maximum d'abrasion résultant de l'équation (1) vaut pour l'acier au carbone non allié. Pour les aciers à $2,5 \%$ de $\mathrm{Cr}$, on admet, pour $f(\vartheta)$, le quart de la valeur. Pour l'acier à $13 \%$ de $\mathrm{Cr}$ on pose $f(\vartheta)=0$.

Les documents indiqués ci-dessus correspondent aux procédés exposés déjà autrefois [1]. Nos expériences et considérations nouvelles montrent qu'on peut s'en tenir vraiment bien à ce propos qu'il y a lieu, bien entendu, de corriger et de commenter sur certains points.

Des examens des taux d'érosion dus à un courant d'eau pure révèlent un maximum à $150^{\circ} \mathrm{C}$ environ. Nos propres évaluations de comptes rendus d'expertises indiquent au contraire que le taux maximum se situe vers $180^{\circ} \mathrm{C}$ pour un courant de vapeur humide avec $x>0.7$. Cet écart est plausible tout d'abord parce que l'évaluation suivant la formule (1) se rapporte, avec $c$ fictive, à une répartition homogène de la vapeur et de l'eau. Un écoulement approximativement homogène de brouillard régi dans la phase gazeuse par la prépondérance des efforts dus aux tensions de cisaillement et à l'inertie et dans la phase aqueuse par celle des efforts dus aux tensions interfaciales ne peut se concevoir que dans un éloignement important de la paroi. Aux parois se forme un film d'eau qui, si mince qu'il soit, est soumis à l'effet de touage exercée par le courant principal et qui est conditionné essentiellement par des efforts de cisaillement et d'inertie. Comme il ressort de la figure 5 , il est facile de comprendre qu'on associe la vitesse homogène de substitution d'un courant d'eau pure à chaque écoulement de film décrit par une vitesse fictivement homogène. Le rapport entre la vitesse de la vapeur et celle de l'eau, c'est-à-dire le glissement, peut être exprimé de différentes manières où l'épaisseur du film qui joue un rôle décisif, est déterminé surtout par l'humidité relative et par la densité de la vapeur.

Comme les essais le démontrent, le courant biphasé est, en règle générale, affecté d'une chute de pression de 1 à 2 fois plus importante que celle qui résulte de la considération d'un milieu homogène; il est évident qu'à $x=0$ et $x=1$, ce facteur passe à la valeur 1. En mettant sur le même pied les pertes de charge dans le courant biphasé et dans le courant d'eau pure équivalent, on peut constater, sur base d'une interdépendance

$$
\Delta p \sim c^{n}
$$

entre les pertes de charge et la vitesse, une bonne concordance avec des résultats de mesure si on pose l'exposant $n=1$ dans l'étendue des grandes teneurs de vapeur et des densités relativement faibles. Par contre, $n=2$ procure une meilleure concordance pour les faibles teneurs de vapeur et les fortes densités. Mais ces dernières conditions tombent déjà en dehors des étendues où l'érosion - corrosion surgit dans les turbines à vapeur.

Le résultat quantitatif d'évaluation par cette méthode de calcul confirme fort bien, lui aussi, que le taux maximum d'abrasion constaté à $150^{\circ} \mathrm{C}$ pour le courant d'eau se déplace vers $180^{\circ} \mathrm{C}$ pour le courant de vapeur humide. Par contre, ces considérations donnent une concordance moins satisfaisante quant à la directive par laquelle le taux d'abrasion est calculé proportionnellement à la racine de l'humidité suivant l'équation (2). Cet écart indique plutôt que dans l'étendue des courants usuels de vapeur humide $(x>0,7)$, l'humidité n'exerce pas une influence importante sur la vitesse du film d'eau. Il est difficile d'estimer les conséquences qui s'ensuivent pour $f(x)$ car il est certain qu'outre la vitesse du film, l'épaisseur de celui-ci a une importance en vue des phénomènes de diffusion. Supposé la présence de vapeur humide, il est concevable néanmoins que le degré d'humidité relative ait un effet un peu amoindri par rapport à celui qui est à la base du calcul. Une revision des comptes rendus évalués jusqu'à présent montre cependant que $f(x) \sim \sqrt{1-x}$ procure une meilleure correspondance entre les calculs et les constatations que $f(x)=$ const.

Toujours est-il qu'il réclame une vérification si l'interposition de séparateurs d'eau dans les tuyauteries reste encore profitable lorsqu'il sert dans un cas concret à la diminution exclusive du danger d'érosion - corrosion car une certaine humidité résiduelle ne se laisse tout de même pas éliminer. Des considérations théoriques et des expériences relatives aux facteurs dépendant de la géométrie (Fig. 4) n'ont, dans presque tous les cas envisagés, pas soulevé la nécessité de modifier ces mêmes facteurs. C'est ainsi qu'une investigation sur l'opportunité de la mise en application des coefficients de pertes de charge connus de l'hydraulique pour les configurations en question a montré que les facteurs pour les cas 1 à 13 sont consistants jusqu'à ce point que, pour eux, l'expression :

$$
\frac{K_{c}}{\sqrt{\xi \frac{A c}{A s}}}
$$

fournit une valeur à peu près constante de 0,5 lorsqu'on y substitue pour $\xi$ la perte de charge rapportée à la pression dynamique, pour $A_{c}$ la section de passage au courant et pour $A_{s}$ la surface en danger. Par $A_{s}$ on entend par exemple la surface totale de tuyauteries rectilignes et celle du côté sous pression de coudes.

Une série de recherches systématiques effectuées au laboratoire sur des plans de retenue et sur des coudes aux rapports différents de courbure procurait une fort bonne concordance des taux d'abrasion mesurés comparés aux valeurs correspondantes pour $K_{c}$.

De toutes manières il est à conseiller d'être prudent lorsqu'on décide du choix des surfaces en aval qu'on veut encore associer au risque de la forme géométrique. C'est ainsi qu'il ne suffit généralement pas de se limiter à fabriquer seulement les pièces façonnées pour des coudes, dérivations et jonctions d'une qualité de 
matière première répondant aux sollicitations. Il faudrait plutôt prévoir également dans la qualité appropriée la tuyauterie en aval et ce sur une longueur de quelques multiples de son diamètre. Aussi faut-il entendre la valeur $K_{c}=0,04$ pour une tuyauterie rectiligne à partir d'une distance relativement grande d'un point de perturbation.

Pour la construction de turbines à vapeur, le courant dans les garnitures à labyrinthes représentées par les configurations 15 et 16 de la figure 4 a une importance particulière. Les coefficients $K_{c}=0,08$ resp. 0,3 indiqués, se sont avérés généralement un peu trop grands. De fait, on peut s'appuyer sur des expériences plus favorables et il serait plus exact de compter sur $K_{c}=0,05$ resp. 0,2. Aux endroits où l'on constate des abrasions, leur agencement géométrique fournit néanmoins une image claire du mécanisme d'écoulement de ce phénomène. En écoulement fort compressible, la vapeur atteint sa plus grande vitesse aux dernières chicanes d'un peigne d'étanchéité et en conséquence, les abrasions commencent, le cas échéant, en cet endroit. Comme il ressort encore de la figure 6 , il est important de noter que dans les configurations de cette espèce, il n'y a pas que l'endroit face aux pointes de chicanes et le point de barrage sur les chicanes qui sont exposés au danger d'érosion - corrosion mais également les étendues des surfaces de fond des chambres à labyrinthes où le courant secondaire vient buter d'une manière prononcée.

Pour l'influence de la vitesse sur le taux d'abrasion, les méthodes décrites de prévision donnent au-delà de la valeur de seuil $K_{c}$, - dont la grandeur adoptée jusqu'à présent peut être maintenue--, une relation linéaire. Les données qu'on peut puiser dans la littérature technique sont très contradictoires mais se situent néanmoins en moyenne près d'une proportionnalité du taux d'abrasion en rapport avec la vitesse. On ne peut certainement pas exclure des abrasions dans le milieu en repos; elles sont de toute façon insignifiantes au point que, techniquement parlant, elles peuvent être négligées mais il faut se garder de supposer que les espaces morts auxquelles on n'attribue de nom aucune vitesse de passage ne soient pas affectés d'érosion-corrosion lorsqu'on doit y attendre des pulsations. Un exemple est foumi par des traces d'abrasion dans l'espace creux de l'enveloppe d'une soupape de régulation d'admission de vapeur vive (Fig. 7). Les marques à la surface du fond du corps de soupape sont manifestement dues à l'érosion - corrosion qui s'est produite à charge partielle de la machine. L'humidité de la vapeur était certainement inférieure à $0,3 \%$. Pour la voie de calcul, on ne peut pas attendre des vitesses élevées à cet endroit, mais il est concevable, - et des enregistrements de vitesses élevées dans le courant au travers de soupapes en position fortement étranglée l'indiquent instamment -, que des pulsations à très haute fréquence sont à l'origine de cette érosion - corrosion. Que l'érosion - corrosion peut surgir même à des endroits qui sont à peine touchés par le courant, est confirmé par la constatation du phénomène au droit localement très limité de quelques clavettes de rangées d'aubes directrices fabriquées en acier non allié (Fig. 8).

Le choix de matières premières appropriées peut dans tous les cas éviter l'érosion - corrosion. Les aciers à $13 \%$ de $\mathrm{Cr}$ et les aciers $\mathrm{Cr}-\mathrm{Ni}$ austénitiques sont durables parce qu'ils ont une tenue passive envers la vapeur telle qu'elle est conditionnée dans les turbines exploitées à la vapeur saturée. Il est pourtant peu économique de faire appel à ces aciers lorsque d'autres aciers, faiblement alliés, conviennent. Parmi les aciers ferritiques faiblement alliés, celui $10 \mathrm{Cr}$ Mo $910(2,25 \% \mathrm{Cr} ; 1,0 \% \mathrm{Mo})$ a une durée relativement bonne.

La figure 9 représente la partie haute pression d'une turbine à vapeur humide et donne des renseignements sur les matières premières mises en cuuvre. Les aubes et les bandages d'étanchéité sont en acier à $13 \% \mathrm{Cr}$. A l'égard de l'effet d'érosion - corrosion, les faces internes de l'enveloppe intérieure sont sujettes aux mêmes conditions que les aubes. C'est pourquoi la possibilité se présente de prévoir de l'acier faiblement des aubes en y rapportant des bagues en acier à $13 \% \mathrm{Cr}$ ou en y appliquant un chargement par soudage. Des réalisations semblables ont fait leurs preuves. Toutefois, l'enveloppe intérieure complète est désormais exécutée en acier coulé à $12 \% \mathrm{Cr}(\mathrm{G}-\mathrm{X} 8 \mathrm{Cr} \mathrm{Ni} 12)$ pour des raisons d'économie. L'arbre est en acier à $3 \% \mathrm{Ni}-$ $1,5 \% \mathrm{Cr}$ (26 Ni Cr Mo V 115). La résistance à l'érosioncorrosion de cet acier a revenu est suffisante dans la zone des aubes, mais dans celle des garnitures extrêmes d'occlusion de l'arbre où la vitesse et l'humidité de la vapeur sont plus élevées on a prévu une soudure à superposition d'acier à $13 \% \mathrm{Cr}-4 \% \mathrm{Ni}(\mathrm{X} 3 \mathrm{Cr} \mathrm{Ni} 134)$.

L'enveloppe extérieure et la conduite de passage à la partie basse pression sont en aciers à $2,25 \% \mathrm{Cr}$ $1 \%$ Mo (GS-18 Cr Mo 910 resp. $10 \mathrm{Cr}$ Mo 910). Les joints d'assemblage et l'étendue des garnitures d'occlusion du passage de l'arbre de l'enveloppe extérieure reçoivent en outre une recharge de X3 $\mathrm{Cr} N \mathrm{Ni} 134$ par soudage.

La figure 10 représente la partie basse pression. En vue de combattre le danger d'érosion-corrosion, on n'introduit que quelques anneaux distincts en acier à $2,25 \mathrm{Cr}-1 \%$ Mo (GS-18 Cr Mo 910) dans l'enveloppe intérieure. Pour le reste et pourvu qu'il y ait surchauffe intermédiaire, on utilise les mêmes matières premières que dans les turbines conventionnelles, c'est-à-dire des aciers à $13 \% \mathrm{Cr}$ pour les aubes et les bandages d'étanchéité et un acier à $3,5 \% \mathrm{Ni}-1,5 \% \mathrm{Cr}(26 \mathrm{Ni} \mathrm{Cr}$ Mo V 145) pour l'arbre.

Ici suit un rapport des constatations faites sur une turbine de $600 \mathrm{MW}$ d'un certain âge déjà et dont la partie haute pression était encore fabriquée en acier coulé GS-C25 non allié (Fig. 11). Après une durée de service de $10000 \mathrm{~h}$ seulement, l'enveloppe était considérablement creusée vers l'étendue de la décharge. Des interventions pour réparation valable étaient prévues seulement à la révision suivante. Pour l'intervalle de $13000 \mathrm{~h}$ de marche, on a mis à l'épreuve sous les conditions d'exploitation et localisées aux surfaces abrasées des éprouvettes d'aciers et de fontes d'acier différents ainsi que des couches projetées de deux alliages métalliques (Fig. 12). Cette période passée, il s'avérait que l'éprouvette en acier non allié St $37-2$ dont la résistance à l'érosion-corrosion est comparable à celle de la matière première de l'enveloppe, était abrasée de plusieurs $\mathrm{mm}$. En opposition à cela, le 


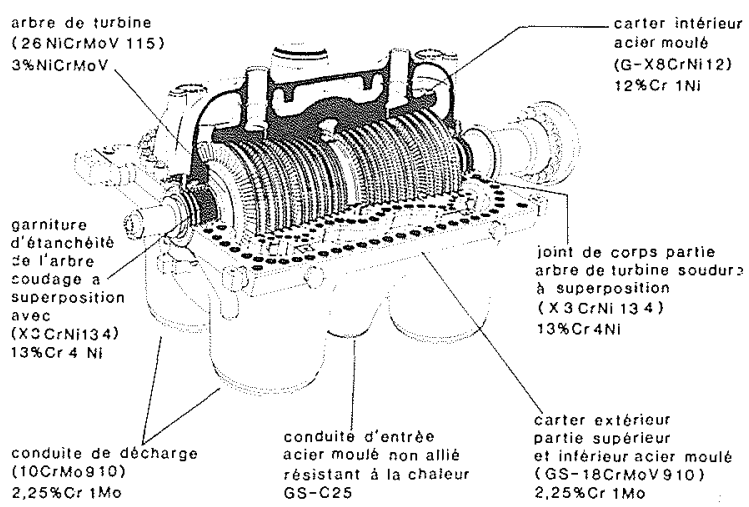

Figure 9 - Choix des matériaux pour turbine HP.

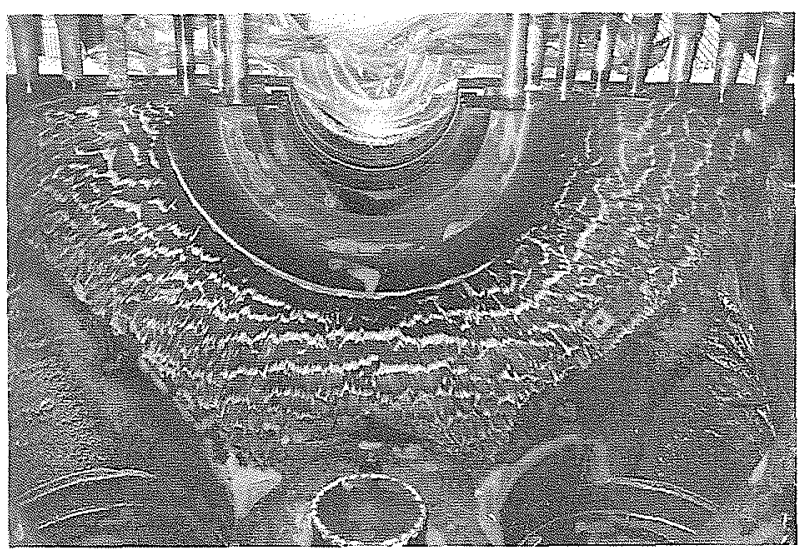

Figure 11 -Erosion-corrosion dans un carter extérieur HP. Matériau : GS-C25, durée de marche : $23239 \mathrm{~h}$.

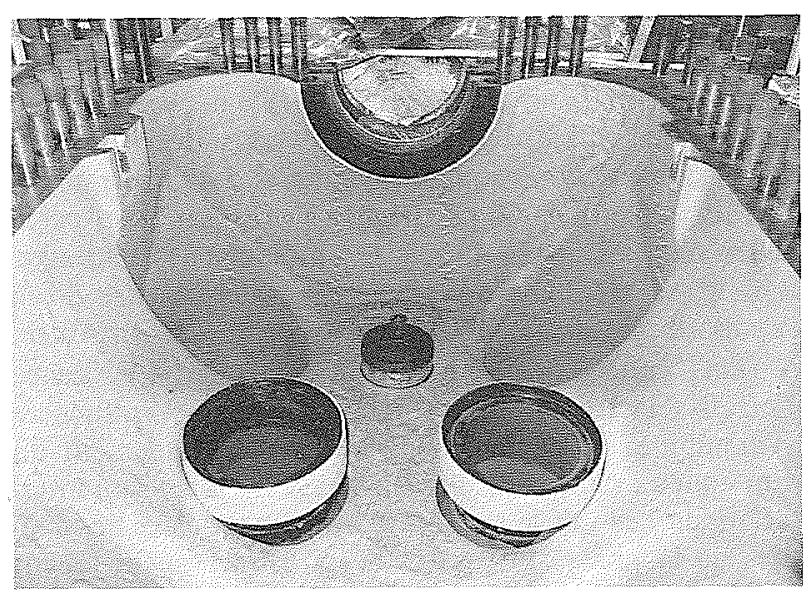

Figure 13 - Rechargement d'un carter extérieur HP usé. Matériau : metcoloy 33 sur GS-C25.

Figure 15 - Erosion-corrosion dans les turbines à vapeur saturée. Délimitation vers l'érosion comme phénomène surtout mécanique.

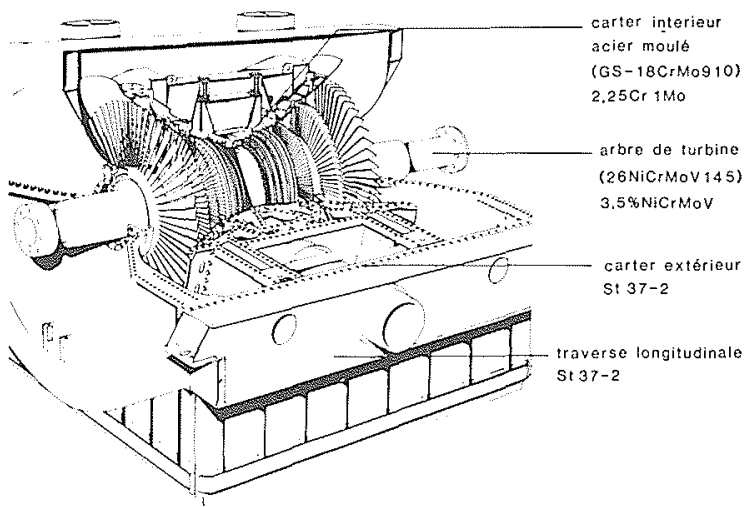

Figure 10 - Choix des matériaux pour turbine BP.

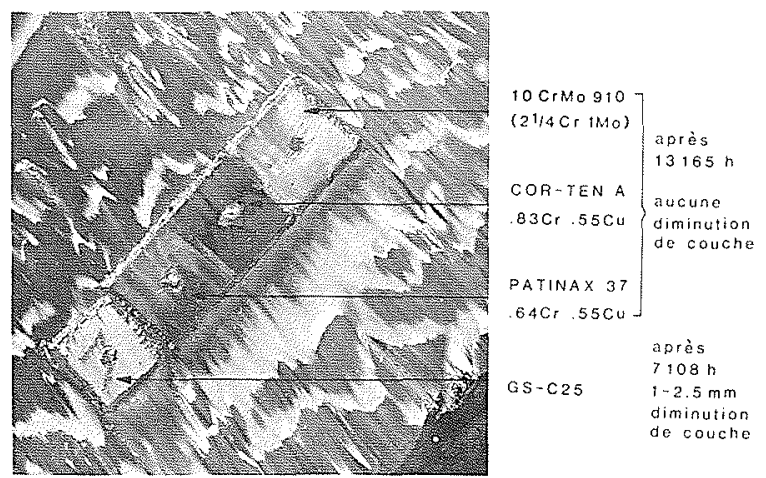

Figure 12 - Comportement de différents matériaux à l'érosioncorrosion.

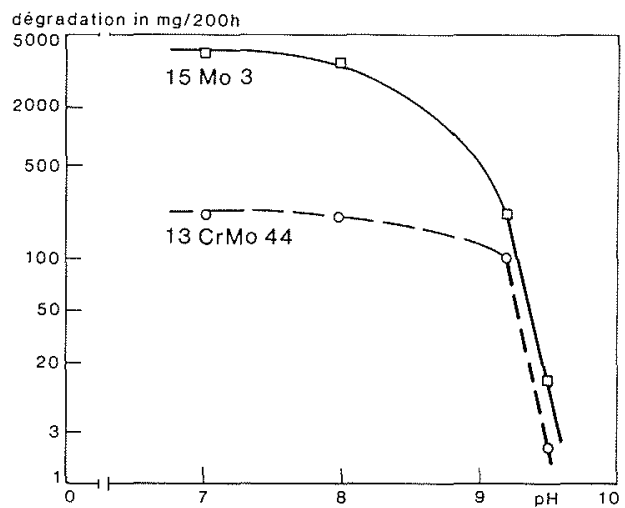

Figure 14 - Influence du pH à la dégradation d'après Heitmann.

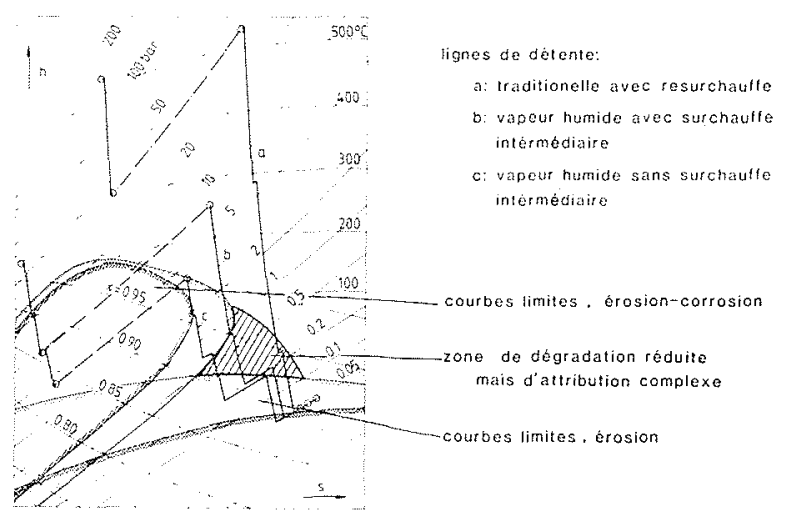


10 Cr Mo 910 et le GS - 18 Cr Mo 910 ainsi que deux autres éprouvettes en acier de construction résistant aux intempéries étaient recouverts d'une couche adhérente de magnétite et ne montraient pas de traces d'érosion-corrosion. Aussi les couches projetées d'alliages métalliques s'étaient très bien tenues. On ne trouvait pas d'abrasion par érosion-corrosion ni rouille par en-dessous. On avait projeté un alliage à base de nickel avec $17 \% \mathrm{Cr}$ et $17 \% \mathrm{Fe}$ (Metcoloy 33 ) et un acier austénitique à $18 \% \mathrm{Cr}, 8,5 \% \mathrm{Mn}$ et $5 \% \mathrm{Ni}$ (Metcoloy 5).

Entretemps, d'excellents résultats d'expériences en durée de $50000 \mathrm{~h}$ sur un groupe turbo-alternateur de $300 \mathrm{MW}$ étaient devenus disponibles. Par précaution, on avait déjà muni au cours de la fabrication le rotor (matériau C 35) et les faces internes de l'enveloppe intérieure (matériau GS-C 25) d'une couche métallique projetée contenant $20 \% \mathrm{Cr}$. En vertu de ces bonnes expériences on a pourvu l'ensemble des surfaces internes de l'enveloppe d'une couche projetée de l'alliage Metcoloy 33 à base de Nickel qui a un coefficient de dilatation thermique analogue à celui du matériau non allié GS-C 25 (Fig. 13). La couche métallique projetée dans cette enveloppe est maintenant mise en cuvre pendant $45000 \mathrm{~h}$ et elle se trouve dans un état remarquablement intact.

Le réglage de la valeur du $\mathrm{pH}$ permet également d'intervenir dans l'effet de l'érosion-corrosion (Fig. 14). C'est une chose connue des essais dans les milieux monophasés que l'abrasion de matière par érosioncorrosion aux aciers de construction faiblement alliés se réduit d'une manière très vive [2] lorsque la valeur du $\mathrm{pH}$ dépasse 9,5. Un effet semblable s'obtient également par des dosages croissants d'oxygène.

Lors de l'alcalinisation du circuit de vapeur d'eau à l'aide des moyens employés habituellement dans les centrales électriques, - l'ammoniac, la morpholine ou l'hydrazine -, il y a lieu de prendre en consideration que pour obtenir la valeur 9,5 de $\mathrm{pH}$, des concentrations très différentes sont requises suivant le produit alcaligène. Comme dans les parties d'une installation sollicitée par la vapeur saturée la phase aqueuse est déterminante pour l'érosion-corrosion, ce n'est en outre que la seule valeur du $\mathrm{pH}$ s'établissant localement dans cette phase aqueuse suivant un certain coefficient de répartition qui est d'une importance décisive.

La morpholine et l'hydrazine ont un coefficient de distribution favorable $(<1)$. Ceci signifie que lors de l'alcalinisation au moyen de ces produits, ceux-ci s'installent de préférence dans la phase aqueuse. L'ammoniac, au contraire, a un coefficient de répartition défavorable, nettement $>1$. Malgré le coefficient de répartition favorable, l'utilisation de morpholine présente des désavantages importants. Pour établir une valeur du $\mathrm{pH}>9,5$, il faut avoir recours à des concentrations très élevées d'environ $100 \mathrm{mg} / \mathrm{kg}$. De plus, les échangeurs d'ions à résines dans le poste de déminéralisation sont pollués d'une façon irréversible. Par contre, des quantités économiques d'hydrazine permettent d'obtenir une alcalinisation jusqu'à peu près $\mathrm{pH}=9,5$ dans la phase de condensation.

Cependant, l'alcalinisation à l'ammoniac est plus rentable lorsqu'on envisage d'atteindre une valeur de
pH au-delà de 9,5 dans la phase aqueuse de la vapeur humide. Les rapports défavorables de répartition de l'ammoniac entre les phases vapeur et aqueuse réclament assurément des dosages augmentés d'ammoniac de l'eau alimentaire. Cette concentration élevée d'ammoniac ne peut pas être réalisée lorsque les condenseurs sont munis de tubes en alliage de cuivre car ces alliages sont sensibles à la corrosion par l'ammoniac. Une solution qui offre plus de chances pour résoudre le problème est la mise en œuvre de titane ou de matériaux austénitiques qui ne souffrent pas de cette attaque corrosive.

Jusqu'ici, les réflexions portaient sur l'attribution de l'érosion-corrosion au concours de phénomènes électrochimiques et thermo-hydrauliques. Mais on a indique que son effet d'abrasion peut se manifester, - ne fût-ce que dans une mesure moindre-, en l'absence de courants imposés. Dans ce cas, l'abrasion est déterminée essentiellement par les processus électro-chimiques. Inversement, l'influence mécanique peut aussi être prépondérante et il est donc facile de comprendre qu'on est incité à présumer une relation entre l'érosioncorrosion telle que définie jusqu'à présent et l'érosion par impact de l'égouttage car une corrosion dite pure parce qu'agissant de par des influences mécaniques est toujours liée à une corrosion [2]

Dans la figure 15 on a représenté sur le diagramme de Mollier pour la vapeur d'eau les étendues typiques dans lesquelles on peut habituellement s'attendre aux deux phénomènes d'érosion-corrosion et de corrosion par impact de l'égouttage. Elles se coupent seulement dans une zone qui n'entre généralement pas en ligne de compte pour l'établissement des turbines exploitées à la vapeur humide. Il faut annoncer cependant que des règles très simplifiées sont à la base de cette représentation dont l'imperfection s'exprime par le fait qu'on constate souvent lors de revisions de turbines des marquages ou des abrasions pour lesquels on ne parvient pas à trouver un rattachement net et exclusif à l'un ou l'autre phénomène "classique". Dans ce contexte, il s'agit dans la plupart des cas de constatations faites dans la zone d'admission de vapeur aux dernières rangées d'aubes directrices de parties basse pression et en particulier aussi à l'entrée d'espaces annulaires de soutirages. Il faut les attribuer essentiellement aux gouttes d'eau expulsées des aubes mobiles par la force centrifuge.

Dans cette zone limite, non seulement l'influence d'effets électro-chimiques recule mais les influences hydrauliques se déplacent de la zone d'écoulement du film aqueux dans celle de l'égouttage où la tension interfaciale de l'eau et le rapport extrêmement élevé des densités de la phase aqueuse et de la phase vapeur jouent un rôle important. Dans cette zone transitoire, c'est de plus en plus la dureté du matériau qui vient se mettre au premier plan à la place de ses composants alliés et ce pour devenir la grandeur prépondérante aux étages finaux.

En ce moment, il n'y a pas un besoin urgent de poser une règle commune englobant la description des deux phénomènes car les abrasions constatées dans cette zone de transition sont minimales mais une réflexion simultanée sur ces phénomènes pourrait sans doute contribuer à leur meilleure compréhension. 
Les expériences acquises au cours des dernières années en matière d'érosion-corrosion dans des turbines exploitées à la vapeur humide ont été un appui pour le bien-fondé des dispositions prises jusqu'à présent.

Par rapport aux attentes, les écarts constatés à l'occasion des revisions de turbines sont d'une portée très limitée. Les corrections qui s'en ensuivent pour les méthodes de prédiction ont été commentées. On ne peut encore s'attendre à des modifications fondamentales dans le choix des matières premières que si l'eau alimentaire est conditionnée de sorte que l'alcalinité de l'eau se dégageant dans la turbine est changée d'une manière essentielle.

\section{Références}

[1] KELLER H. - Problèmes de corrosion et d'érosion dans les turbines à vapeur saturée: Journées Internationales d'Etude des Centrales Electriques Modernes. A.I.M. Liège 1978.

[2] HEITMANN H.G. - Korrosion auf der Wasser - und Dampfseite: Jahrbuch der Dampferzeugungstechnik, 4. Ausgabe 1980/81, Vulkan-Verlag/Essen

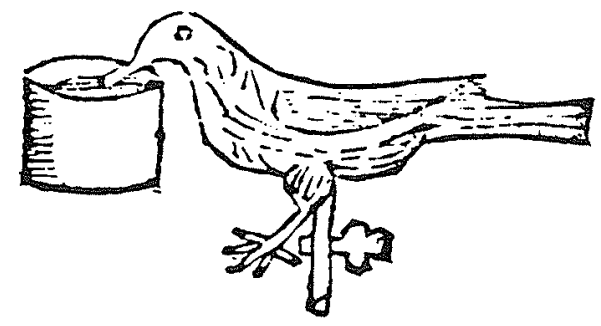




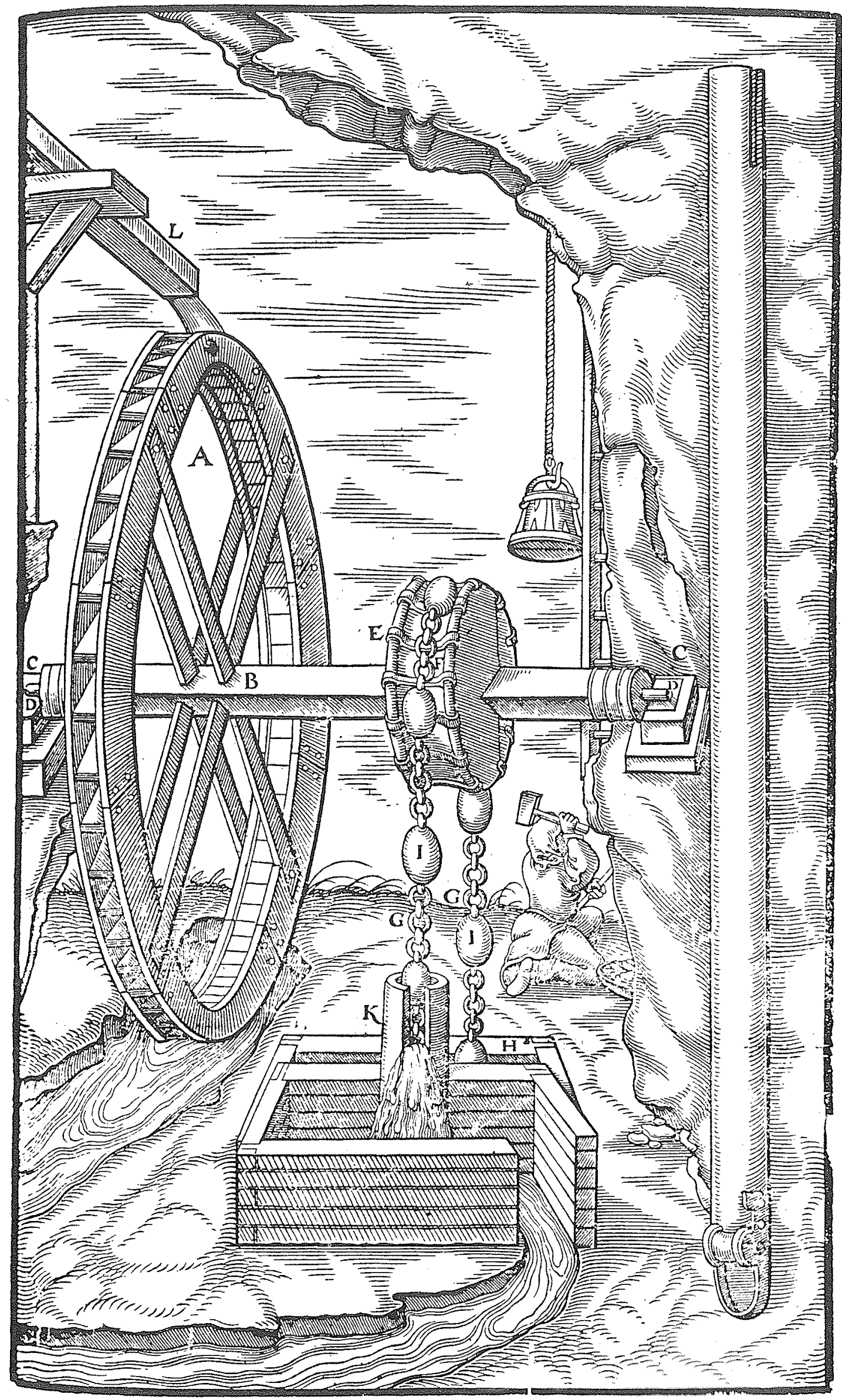

